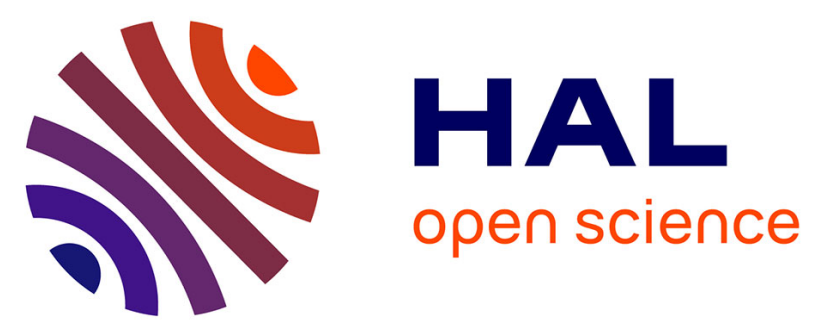

\title{
Optimization of a photopolymerizable material based on a photocyclic initiating system using holographic recording
}

\author{
Ahmad Ibrahim, Christian Ley, Xavier Allonas, Olga Tarzi, Aurélie Chan \\ Yong, Christiane Carré, Raymond Chevallier
}

\section{To cite this version:}

Ahmad Ibrahim, Christian Ley, Xavier Allonas, Olga Tarzi, Aurélie Chan Yong, et al.. Optimization of a photopolymerizable material based on a photocyclic initiating system using holographic recording. Photochemical \& Photobiological Sciences , 2012, 11, pp.1682-1690. 10.1039/C2PP25099C . hal00920012

\section{HAL Id: hal-00920012 \\ https://hal.science/hal-00920012}

Submitted on 17 Jan 2014

HAL is a multi-disciplinary open access archive for the deposit and dissemination of scientific research documents, whether they are published or not. The documents may come from teaching and research institutions in France or abroad, or from public or private research centers.
L'archive ouverte pluridisciplinaire HAL, est destinée au dépôt et à la diffusion de documents scientifiques de niveau recherche, publiés ou non, émanant des établissements d'enseignement et de recherche français ou étrangers, des laboratoires publics ou privés. 


\title{
Photochemical \&

\section{Optimization of a photopolymerizable material based on a photocyclic initiating system using holographic recording $\dagger$}

\author{
A. Ibrahim, ${ }^{a}$ C. Ley, ${ }^{a}$ X. Allonas, ${ }^{* a}$ O. I. Tarzi, ${ }^{a}$ A. Chan Yong, ${ }^{b, c, d}$ C. Carré ${ }^{b, d}$ and R. Chevallier ${ }^{e}$ \\ Received 12th April 2012, Accepted 21st May 2012 \\ DOI: 10.1039/c2pp25099c
}

A set of photoinitiating systems (PIS) for free radical photopolymerization was studied using time-resolved spectroscopic experiments, real-time FTIR and holographic recording. It is shown that the efficiency of the photoinitiating system can be drastically increased when a redox additive is added to the conventional dye/coinitiator system by virtue of photocyclic behaviour. The homogeneous photopolymerization process was found to reach a fast vitrification, limiting the conversion at about $55 \%$. By contrast, holographic recording underlines the differences in photoinitiating system reactivity, allowing diffraction efficiencies close to unity for the most reactive PIS.

\section{Introduction}

Light induced polymerization reactions are employed in quite different technical applications that have become beneficial to humans. These applications include microelectronics, information technologies, optical fibers, dental materials, printing inks, paints and varnishes. ${ }^{1-3}$ In other words, various kinds of polymers can be synthesized by light-induced chemical processes, a technique commonly denoted by the term photopolymerization. A key component of this process is the photoinitiating system, which is responsible for the absorption of light and its conversion into chemical energy.

Among these applications, photopolymeric materials have been revealed to be the best choice for holographic recording, promoting holography as a compelling choice for next-generation data storage needs. ${ }^{4}$ In holography and display devices, photopolymers were developed primarily for the display of single images due to the refractive index variations or relief profiles generated by an optical interference pattern. ${ }^{5,6}$ Compared to inorganic photorefractive materials, photopolymers have much higher sensitivity due to chemical amplification effects, large dynamical range and high versatility of the formulations. Additionally, this is a relatively low cost one step process. ${ }^{7-11}$ Data storage applications require materials corresponding to more critical properties, such as hologram

\footnotetext{
${ }^{a}$ Laboratory of Macromolecular Photochemistry and Engineering, University of Haute Alsace, ENSCMu, 3 rue Alfred Werner, 68093 Mulhouse,France.E-mail: xavier.allonas@uha.fr;

Fax: + 33 389335014; Tel: +33 389335011

${ }^{b}$ UEB, Université Européenne de Bretagne, France

'Institut Mines-Télécom, Télécom Bretagne, Optics Department, Technopôle Brest Iroise CS 83818, 29238 Brest Cedex 3, France

${ }^{d}$ CNRS, UMR 6082 FOTON, Enssat, 6 rue de Kerampont, BP 80518, 22305 Lannion, France

eInstitut Mines-Télécom, Mines de Douai, 941 Rue Charles Bourseul, 59508 Douai Cedex, France

$\dagger$ This article is published as part of a themed issue in honour of JeanPierre Desvergne on the occasion of his 65th birthday.
}

shrinkage or stability, and different classes of recording polymer materials have been studied extensively in the past decade. In this area, epoxy resin associated to a triacrylic monomer, ${ }^{12}$ polymers based on mixture of polyvinylalcohol and acrylamide or sodium acrylate, ${ }^{5}$ nanocomposite materials in the form of an acrylamidebased polymer containing zeolite nanoparticles ${ }^{13}$ or consisting of an acrylate monomer in poly(methylmethacrylate) doped with $\mathrm{ZrO}_{2}$ or $\mathrm{SiO}_{2}$ nanoparticles ${ }^{14}$ were described.

Tailoring of photopolymeric materials is one specific application of visible light photoinitiating systems (PIS). ${ }^{2,5,15}$ Basically, visible light PIS comprises a dye chosen to absorb at a specific irradiation wavelength and a coinitiator that produces free radicals able to initiate the polymerization reaction. ${ }^{2,3}$ However, these two-component PIS have moderate photopolymerization efficiency, and the addition of a third component was found to be beneficial for improving the performance of these systems. In that case, secondary redox reactions take place after the primary photochemical reaction, and a higher yield of initiating species is obtained. Therefore, three-component photoinitiating systems have been found to be by far more efficient than the corresponding two-component systems. ${ }^{2,16-19}$ However, the actual effects of coinitiator concentration, monomer structure, resin viscosity and redox potentials are still unclear.

Recently, the efficiency of three-component PIS based on pyrromethene dyes for free radical polymerization was discussed. It was shown that the photopolymerization efficiency of the three-component PIS containing an amine and a triazine derivative together with the pyrromethene dye is higher than that of the corresponding two-component systems, and the effect of the relative concentration of the coinitiator was detailed..$^{20,21}$

Pyrromethene dyes present encouraging properties as efficient photosensitizers. Their photophysical properties are well known and they show attractive laser performance. ${ }^{22-27}$ Strong absorption and fluorescence bands in the visible region were observed for this family of dyes. ${ }^{21,28}$ In addition, their high laser efficiencies are not only due to their high emission quantum yield 
$\left(\varphi_{\mathrm{f}}>0.8\right)$, but also to the low probability of triplet formation and triplet-triplet absorption over the laser spectral region, ${ }^{27,29-32}$ leading to high photostability. ${ }^{33-37}$ Thus, many applications were developed with these compounds in various fields, such as optoelectronics, ${ }^{38,39}$ solid-state dye lasers, ${ }^{27,40-42}$ fluorescent probes in biochemical procedures, ${ }^{43,44}$ visible laser imaging ${ }^{29,42}$ and photoinitiators for free radical photopolymerization. ${ }^{45,46}$ In addition, the laser efficiency and photostability of pyrromethene dyes have been widely studied in hosts like methylmethacrylatebased polymers, hybrid sol-gel materials and solutions. ${ }^{34,35}$ Moreover, these organic dyes have been used as fluorescent compounds in a polymeric host for $3 \mathrm{D}$ optical data storage. ${ }^{47,48}$

In this work, we used a photopolymerizable resin, optimized as an efficient holographic recording medium, to probe the reactivity of photoinitiating systems based on a pyrromethene dye (EMP). We investigated the combination of three different coinitiators considering an amine (EDB) as the electron donor and an electron acceptor, which could be either a triazine derivative (TA) or an iodonium salt (I250). Time resolved spectroscopy experiments allow us to describe the behaviour of the different photoinitiating systems. It is found that the dye is invested in a photocyclic reaction in which each photon absorbed leads to two initiating radicals. Accordingly, a mechanism is proposed in full agreement with our previous works. ${ }^{20,21}$ Real-time FTIR spectroscopy (RT-FTIR) was used to characterize the photopolymerization kinetics of these systems by following the evolution of monomer conversion during the photopolymerization reaction. The influence of the experimental conditions (irradiation intensity and irradiation time) on the performance of these PIS using holographic recording was also examined by recording the diffraction efficiency as a function of irradiation time. This kinetic study of grating formation is discussed according to the RT-FTIR photopolymerization experiments. It is shown that holographic recording reveals interesting features of PIS reactivity, which are levelled off when studied by RT-FTIR.

\section{Experimental}

\section{Chemicals}

2,6-Diethyl-1,3,5,7,8-pentamethylpyrromethene $\mathrm{BF}_{2}$ complex (EMP) was purchased from Exciton. Ethyl-4-(dimethylamino)benzoate (EDB) was obtained from Aldrich, 2-(4-methoxy-1naphthyl)-4,6-bis(trichloromethyl)-1,3,5-triazine (TA) and (4methylphenyl)[4-(2-methylpropyl)phenyl]-iodonium hexafluorophosphate (I250) were gifts from PCAS (France) and Ciba Spa. (Switzerland), respectively. Their chemical structures are given in Scheme 1.
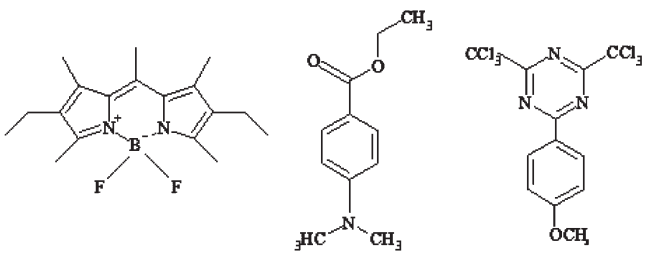

EMP
EDB

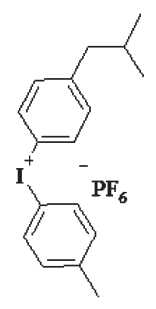

1250

\section{Photopolymerizable resin}

The reactive resin was a liquid mixture of different monomers:

- $30 \mathrm{wt} \%$ of a hexafunctional aliphatic urethane acrylate oligomer (Ebecryl 1290, Cytec) acting as primary oligomer;

- $15 \mathrm{wt} \%$ of 1,1,1,3,3,3-hexafluoroisopropyl acrylate and $15 \mathrm{wt} \%$ of vinyl neononanoate, both from Aldrich. The vinyl ester monomer is known to copolymerize very easily with acrylic monomers. The partial fluorination of host polymer matrices resulted in improved optical properties and better defined morphologies; ${ }^{49,50}$

- $4.6 \mathrm{wt} \%$ of $N$-vinyl pyrrolidinone (Aldrich), which is a standard reactive additive introduced in phopolymerizable systems $;{ }^{51}$

- $4.6 \mathrm{wt} \%$ of trimethylolpropane tris(3-mercaptopropionate) (Aldrich), which is a trifunctional thiol able to increase the photopolymerization rate in air and leading to higher monomer conversions. $^{51}$

The choice of the formulation was governed by earlier experiments performed in the field of visible curable systems and the use of fluorinated acrylate monomers for the recording of holographic polymer-dispersed liquid crystals (LC) transmission gratings. 52,53

For all experiments, the photoinitiating system was introduced in the resin in the following weight ratio: $0.2 \mathrm{wt} \%$ of dye, $0.6 \mathrm{wt} \%$ of electron donor and $1 \mathrm{wt} \%$ of electron acceptor.

\section{Cyclic voltammetry}

Redox potentials were measured by cyclic voltammetry using a potentiostat (Princeton Applied Research 263A) at a scan rate of $1 \mathrm{~V} \mathrm{~s}^{-1}$ in acetonitrile, with platinum as both working and auxiliary electrodes, and a saturated calomel reference electrode $(\mathrm{KCl}$ in methanol). Measurements were performed in acetonitrile using $0.1 \mathrm{M}$ of tetrabutylammonium hexafluorophosphate (Aldrich) as supporting electrolyte. The samples were bubbled with argon for 20 minutes prior to the analysis. Ferrocene was used as a standard. ${ }^{54}$

\section{RT-FTIR measurements}

The study of photopolymerization kinetics was carried out by real-time FTIR (RT-FTIR) technique using a Vertex 70 FTIR spectrometer (Bruker Optik), equipped with a MCT detector working in the rapid scan mode. This allows an average of 4 scans per s collection rate using a resolution of $4 \mathrm{~cm}^{-1}$. The IR spectra were then recorded during sample irradiation using a green laser diode emitting at $532 \mathrm{~nm}$ (Roithner Lasertechnik, $50 \mathrm{~mW}$ ), which was adapted to the FTIR spectrometer by means of a light guide. The irradiation intensity was adjusted at $25 \mathrm{~mW} \mathrm{~cm}^{-2}$ on the sample. To prevent the diffusion of oxygen into the sample during the irradiation, experiments were carried out by laminating the resin between two polypropylene films and two $\mathrm{CaF}_{2}$ windows. The thickness of the sample was adjusted using a $25 \mu \mathrm{m}$ teflon spacer. The spectra were recorded between 600 and $3900 \mathrm{~cm}^{-1}$.

The kinetics of the polymerization were measured by following the disappearance of the $\mathrm{C}=\mathrm{C}$ bond stretching signal at $1637 \mathrm{~cm}^{-1}$. The degree of conversion is directly related to the 
decrease of peak area at $1637 \mathrm{~cm}^{-1} A_{1637}$, and was calculated according to:

$$
C(\%)=\frac{\left(A_{1637}\right)_{0}-\left(A_{1637}\right)_{t}}{\left(A_{1637}\right)_{0}} \times 100
$$

where $\left(A_{1637}\right)_{0}$ and $\left(A_{1637}\right)_{t}$ are the area of the IR absorption band at $1637 \mathrm{~cm}^{-1}$ of the sample before exposure and at time $t$ respectively. A good estimate of the maximum rate of conversion $R_{\mathrm{p}}^{\prime}$ was determined by the slope of the conversion kinetics at the inflection point. The conversion rate $R_{\mathrm{p}}^{\prime}$ is linearly related to the rate of polymerization $R_{\mathrm{p}}$ by $R_{\mathrm{p}}^{\prime}=R_{\mathrm{p}} \times 100 /\left[\mathrm{M}_{0}\right]$ where $\left[\mathrm{M}_{0}\right]$ is the initial monomer concentration (i.e. before exposure). According to this linear relationship, the values of conversion rate $R_{\mathrm{p}}^{\prime}$ will be reported in this work. ${ }^{55}$

\section{Spectroscopy measurements}

Laser flash photolysis experiments (LFP) were carried out exciting at $532 \mathrm{~nm}$ with a nanosecond Nd-YAG laser (Powerlite 9010, Continuum), operating at $10 \mathrm{~Hz}$. The transient absorption analysis system (LP900, Edinburgh Instruments) uses a $450 \mathrm{~W}$ pulsed Xe arc lamp, a Czerny-Turner monochromator, a fast photomultiplier, and a transient digitizer (TDS 340, Tektronix). ${ }^{56}$ The instrumental response was about $7 \mathrm{~ns}$. The observation wavelength is indicated in each case. Experiments were performed in acetonitrile under Ar bubbling.

A FluoroMax-4 (Horiba, Jobin-Yvon) spectrofluorimeter coupled with Time-Correlated Single-Photon Counting (TCSPC) accessory was used to measure steady-state fluorescence spectra and singlet excited state lifetimes. NanoLEDs were used as pulsed excitation source leading to a time resolution of around 200 ps. The measurements were performed in acetonitrile solutions under argon bubbling at room temperature.

The quenching rate constants $k_{\mathrm{q}}$ of the excited states were obtained according to the Stern-Volmer analysis where the reciprocal lifetime $\tau^{-1}$ is plotted as a function of quencher concentration:

$$
\frac{1}{\tau}=\frac{1}{\tau_{0}}+k_{\mathrm{q}}[\mathrm{Q}]
$$

where [Q] is the molar concentration of quencher, $k_{\mathrm{q}}$ the quenching rate constant and $\tau_{0}$ the singlet state lifetime in the absence of quencher.

\section{Holographic recording}

The samples were prepared by embedding the photopolymerizable formulation between two glass-substrates. Calibrated glass beads were used as spacers to guarantee the thickness of the system around $20 \mu \mathrm{m}$.

During holographic recording, a sinusoidal light pattern is generated by the interferences of the two incident plane waves and is converted into a modulation of the refractive index in the photopolymerizable matrix. ${ }^{57,58}$ The two incident s-polarized beams were of equal intensity, corresponding to a total power density of 4 or $25 \mathrm{~mW} \mathrm{~cm}^{-2}$ on the photosensitive sample with a beam diameter of $2.5 \mathrm{~cm}$. When the photopolymerizable system is illuminated by the sinusoidal interference pattern, an inhomogeneous polymerization reaction and dye bleaching take place. ${ }^{57,58}$ Monomer and sensitizer consumption in the bright regions led to concentration spatial gradients of monomer and sensitizer molecules, which, in turn, led to diffusion processes. The coupling between photochemical conversion and mass transport results in regions with various matter densities. A modulation of the refractive index is created, giving rise to volume thick phase gratings with high diffraction efficiencies and low scattering noise. In this work, laminated layers were used to give rise only to a modulation of the refractive index, i.e. to suppress any generation of surface relief. The obtained transmission gratings were recorded with a $514 \mathrm{~nm}$ actinic laser light (Coherent Innova 308C Argon Ion laser). The fringe spacing is adjusted to $c a$. $0.9 \mu \mathrm{m}$ and exposure duration of ca. $90 \mathrm{~s}$. The fact that no chemical post-treatment was needed for this recording medium, allowed the continuous follow up of the process during exposure with an inactinic reading light beam (HeNe laser at $633 \mathrm{~nm}$ ), which is more or less diffracted. The diffraction efficiency at $633 \mathrm{~nm}(\eta)$ was defined by the ratio of the intensity of the first diffraction order to the diffracted plus transmitted light intensities. This measurement instead of the ratio of the diffracted intensity by the incident intensity at $633 \mathrm{~nm}$ was performed in order to rule out Fresnel losses in the determination of the grating diffraction efficiency. The rate of grating formation $R_{\eta}$ is calculated as:

$$
R_{\eta}=\frac{\mathrm{d} \eta}{\mathrm{d} t}
$$

\section{Mechanistic studies of the PIS}

The photophysical and electrochemical properties of EMP are given in Table 1.

According to the literature data and to the redox properties of the pyrromethene dye and the coinitiators used, a photoinduced electron transfer reaction is expected between the singlet excited states of the EMP dye and the coinitiators. ${ }^{20,21,28}$ The values of the Gibbs free energy change $\Delta G_{\text {et }}$ for photoinduced electron transfer is given by the Rehm-Weller equation: ${ }^{59}$

$$
\Delta G_{\mathrm{et}}=E_{\mathrm{ox}}-E_{\mathrm{red}}-E *+C
$$

\begin{tabular}{|c|c|c|c|c|}
\hline & $\mathrm{EMP}^{a}$ & $\mathrm{EDB}^{b}$ & $\mathrm{TA}^{b}$ & $\mathrm{I} 250^{b}$ \\
\hline$\lambda_{\max }(\mathrm{nm})$ & 515 & & & \\
\hline$\lambda_{\max }(\mathrm{nm})$ & 536 & & & \\
\hline$\varepsilon_{\max }\left(\mathrm{M}^{-1} \mathrm{~s}^{-1}\right)$ & 70400 & & & \\
\hline$E_{\mathrm{S}}\left(\mathrm{kcal} \mathrm{mol}^{-1}\right)$ & 54.6 & & & \\
\hline$\tau_{\mathrm{f}}(\mathrm{ns})$ & 6.8 & & & \\
\hline$\varphi_{\mathrm{f}}$ & 0.85 & & & \\
\hline$E_{\mathrm{T}}\left(\mathrm{kcal} \mathrm{mol}^{-1}\right)$ & 40.2 & & & \\
\hline$\tau_{\mathrm{T}}(\mu \mathrm{s})$ & 33 & & & \\
\hline$E_{\mathrm{ox}}(\mathrm{V} / \mathrm{SCE})$ & 1.07 & 1.07 & & \\
\hline$E_{\text {red }}(\mathrm{V} / \mathrm{SCE})$ & -1.24 & & -0.99 & -0.82 \\
\hline
\end{tabular}

Table 1 Photophysical and electrochemical properties of EMP, EDB, TA and I250 
where $E_{\mathrm{ox}}$ and $E_{\text {red }}$ are the half-wave oxidation and reduction potentials for the donor and the acceptor, respectively. $E^{*}$ stands for the energy of the excited state. The Coulombic term $C$ is usually neglected in polar solvents.

The calculated Gibbs free energy $\Delta G_{\text {et }}$ for the different EMP/ coinitiator systems are gathered in Table 2 . The data indicate that the intermolecular electron transfer process is not favourable in the ground state, a fact which rules out any dark reaction. Positive values of $\Delta G_{\text {et }}$ are also obtained for the reaction of the triplet excited state of EMP $\left({ }^{3} \mathrm{EMP}\right)$ and all coinitiators, indicating that the photoinduced electron transfer process is not thermodynamically favourable from ${ }^{3} \mathrm{EMP}$.

More interestingly, the $\Delta G_{\text {et }}$ values between the singlet excited state of EMP ( ${ }^{1}$ EMP) and all coinitiators are negative. As EMP is known to exhibit a high fluorescence quantum yield $\left(\varphi_{\mathrm{f}}=0.85\right.$ in acetonitrile ${ }^{28}$ ), the photoreactivity of EMP is expected to occur predominantly from the singlet excited state ${ }^{1}$ EMP.

The values of the ${ }^{1}$ EMP quenching rate constants $k_{\mathrm{q}}$ with the different coinitiators were determined by TCSPC ( $c f$. Table 3 ). As can be seen, TA leads to a quenching rate constant of $k_{\mathrm{q}}^{\mathrm{TA}}=1.3 \times 10^{10} \mathrm{M}^{-1} \mathrm{~s}^{-1}$, which is close to the diffusion limit in acetonitrile $\left(2.0 \times 10^{10} \mathrm{M}^{-1} \mathrm{~s}^{-1}\right)$. A comparable high quenching rate constant value was obtained for I250 $\left(9.0 \times 10^{9} \mathrm{M}^{-1} \mathrm{~s}^{-1}\right)$. The quenching rate constant obtained for EDB is significantly lower (i.e. $4.5 \times 10^{8} \mathrm{M}^{-1} \mathrm{~s}^{-1}$ ), in line with the calculated $\Delta G_{\mathrm{et}}$ values (Table 2).

Pseudo-first order reaction rates (i.e. $k_{\mathrm{q}} \times$ [Coinitiator]) were calculated (Table 3) at coinitiator concentrations found in the resin formulation, namely: $[\mathrm{EDB}]=1.85 \times 10^{-2} \mathrm{M},[\mathrm{TA}]=$ $5.7 \times 10^{-3} \mathrm{M}$ and $[\mathrm{I} 250]=1.75 \times 10^{-2} \mathrm{M}$. As can be seen, the reaction rate with EDB is 10 or 100 times lower than those with TA or 1250 , respectively. These data indicate that the ${ }^{1}$ EMP reacts first with the electron acceptor (TA or I250) in the case of three component systems.

Fig. 1 shows the EMP photobleaching signal at $510 \mathrm{~nm}$ after addition of TA or I250 and after laser pulse excitation at $532 \mathrm{~nm}$. As can be seen, the photoinduced electron transfer between ${ }^{1}$ EMP and the acceptors leads to long-lived species and no ground state recovery is observed: this is attributed to the formation of $\mathrm{EMP}^{\cdot+}$, which does not recombine within the experimental time window. The fast cleavage of $\mathrm{TA}^{\cdot-}$ or $\mathrm{I} 250$ prevents

Table 2 Gibbs free energy $\left(\Delta G_{\mathrm{et}}\right)$ values for ground and excited states electron transfer reactions of EMP/coinitiator systems

\begin{tabular}{llll}
\hline$\Delta G_{\text {et }}(\mathrm{eV})$ & Ground state & Singlet state & Triplet state \\
\hline EDB & 2.31 & -0.06 & 0.57 \\
TA & 2.06 & -0.31 & 0.32 \\
I250 & 1.89 & -0.48 & 0.15 \\
\hline
\end{tabular}

Table $3 \quad{ }^{1}$ EMP quenching rate constants $k_{\mathrm{q}}$ and corresponding pseudofirst order reaction rates with the different coinitiators studied

\begin{tabular}{lll}
\hline Coinitiator & $k_{\mathrm{q}}\left(\mathrm{M}^{-1} \mathrm{~s}^{-1}\right)$ & $k_{\mathrm{q}} \times[$ Coinitiator $]\left(\mathrm{s}^{-1}\right)$ \\
\hline EDB & $4.5 \times 10^{8}$ & $8.3 \times 10^{6}$ \\
TA & $1.3 \times 10^{10}$ & $7.4 \times 10^{7}$ \\
I250 & $9.0 \times 10^{9}$ & $1.6 \times 10^{8}$
\end{tabular}

any back electron transfer, a fact which increases the quantum yield of radical formation. Unfortunately, $\mathrm{EMP}^{++}$cannot be detected, probably due to the low absorption properties of this transient. $^{28,30}$ Subsequent addition of EDB in the solution leads to a reduction reaction of $\mathrm{EMP}^{{ }^{+}}$by EDB, yielding to the recovery of the dye in the ground state and the formation of the radical cation $\mathrm{EDB}^{+}{ }^{+}$. This is clearly highlighted in Fig. 1, which shows that the photobleaching of EMP by TA and I250 is lowered when increasing the amount of added EDB. The observation of this secondary reaction between $\mathrm{EMP}^{++}$and EDB is in line with the corresponding free energy change, which could be calculated as:

$$
\Delta G_{\mathrm{et}}=E_{\mathrm{ox}}^{\mathrm{EDB}}-E_{\mathrm{ox}}^{\mathrm{EMP}}=0
$$

taking into account that $E_{\text {red }}^{\mathrm{EMP}++}=E_{\mathrm{ox}}^{\mathrm{EMP}}$. On the basis of a conventional Rehm-Weller model, one can assume a corresponding rate constant of $10^{9} \mathrm{M}^{-1} \mathrm{~s}^{-1}$, which leads to a lifetime of about $100 \mathrm{~ns}$ for the radical cation at a EDB concentration of $0.01 \mathrm{M}$. The radical cation $\mathrm{EDB}^{++}$created during this reaction undergoes a deprotonation reaction, which yields the formation of a second initiating radical.

The proposed mechanisms for the three component systems based on EMP/EDB/TA and EMP/EDB/I250 are shown in Scheme 2. In a first step, a photoinduced electron transfer process takes place from ${ }^{1}$ EMP to the electron acceptor, yielding to the formation of the radical cation of the dye $\left(\mathrm{EMP}^{\cdot+}\right)$ and the
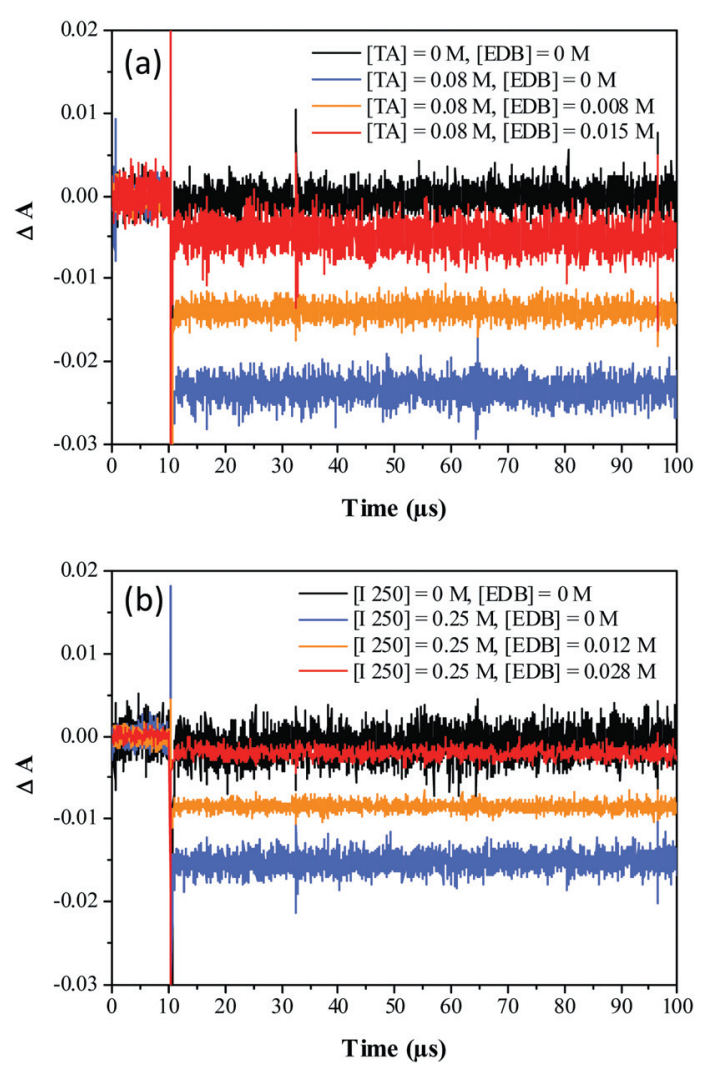

Fig. 1 EMP photobleaching signal measured at $510 \mathrm{~nm}$ in acetonitrile solution under Ar bubbling: effect of EDB addition on (a) EMP/TA solution and (b) EMP/I250 solution. Irradiation at $532 \mathrm{~nm}$. 


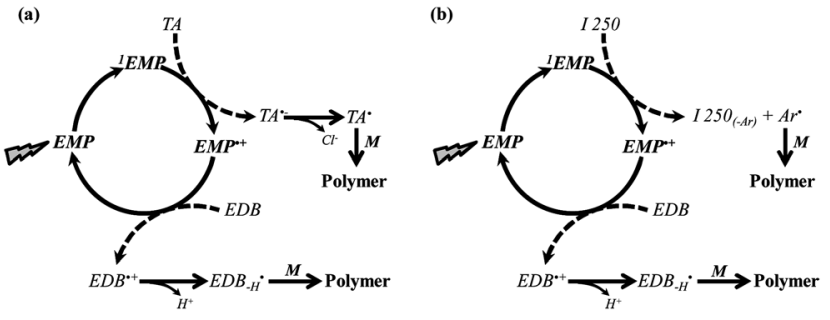

Scheme 2

corresponding radical anion $\mathrm{TA}^{\cdot-}$ of triazine or the reduced form of the iodonium salt.

Then, for both acceptors, a fast cleavage occurs to produce the initiating triazinyl radical $\left(\mathrm{TA}^{*}\right)$ or an aryl radical $\left(\mathrm{Ar}^{*}\right)$ for I250. ${ }^{2,3}$ In a second step, the reaction of $\mathrm{EMP}^{+{ }^{+}}$with EDB leads to the production of a second radical. Moreover, the dye is recovered and is thus available for being invested in further photoreaction. Ideally, the dye is recovered as long as both donor and acceptor are present in the medium, leading to two initiating radicals formed per absorbed photon. Obviously, the efficiencies of the different reactions involved in such photocyclic initiating systems (PCIS) are lower than $100 \%$, the dye is not completely recovered and there are less than two radicals produced by a cycle. The actual quantum yields depend on coinitiator concentrations, reaction rate constants and lifetimes of excited states and radicals. Nevertheless, PCIS have higher reactivity than two components PIS.

\section{Photopolymerization experiments}

\section{EMP/EDB/TA systems}

The evolution of the conversion with irradiation time for EMP/EDB/TA system are displayed in Fig. 2, and the corresponding data are collected in Table 4. The two-component photoinitiating system EMP/EDB shows the lowest conversion rate $R_{\mathrm{p}}^{\prime}$ and final conversion with values around $0.8 \mathrm{~s}^{-1}$ and $44 \%$, respectively.

In addition, holographic recording experiments reveal that this system is not able to give rise to a significant refractive index modulation, i.e. the diffraction efficiency value $\eta$ is zero (cf. Fig. 3(a)). It seems that the minimum energy threshold is higher for holographic recording than for homogeneous polymerization. This behaviour can be rationalized by taking into account the gelation point of the photopolymerized systems. Indeed, homogeneous exposure under RT-FTIR conditions leads to the observation of a small decrease in the double bonds, i.e. some monomer is converted into short polymeric chains: the system EMP/EDB does not lead to any solid film, and indeed the resin, although more viscous, is still liquid after exposure. As a consequence, under holographic recording, the conversion being below the gelation point, these short polymeric chains are slowly diffusing in the dark areas, a fact which vanishes the modulation of the refractive index.

The two-component system based on EMP/TA appears to be more efficient ( $c f$. Fig. 2 and 3(a)). Even if the final conversion is only slightly increased compared to EMP/EDB, the conversion rate is higher by a two-fold factor ( $c f$. Table 4). In that case,
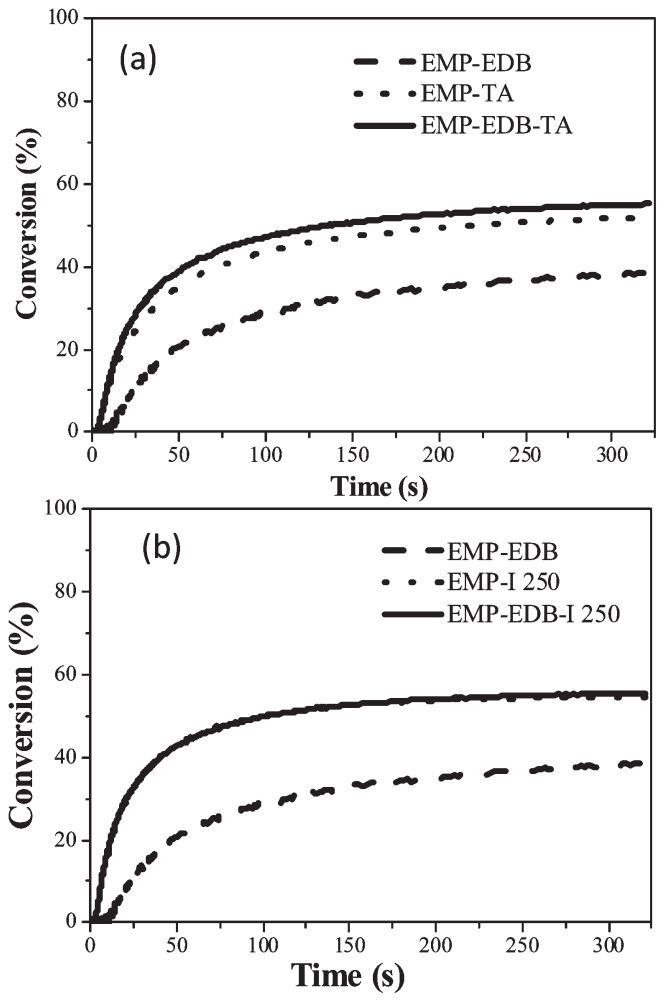

Fig. 2 Conversion curves of the different photoinitiating systems upon green laser exposure at $532 \mathrm{~nm}$ (irradiance $=25 \mathrm{~mW} \mathrm{~cm}^{-2}$ ).

Table 4 Characteristic parameters for the photopolymerization using different EMP based photoinitiating systems. Inhibition time $\left(t_{\text {inh }}\right)$, rate of conversion $R_{\mathrm{p}}^{\prime}\left(\mathrm{s}^{-1}\right)$, maximum conversion $C_{\max }(\%)$, maximum diffraction efficiency $\left(\eta_{\max }\right)$, maximum rate of grating formation $\left(R_{\eta}\right)$.

\begin{tabular}{llllll}
\hline Coinitiators & ${t_{\text {inh }}}^{a}(\mathrm{~s})$ & $R_{\mathrm{p}}{ }^{a}\left(\mathrm{~s}^{-1}\right)$ & $C_{\max }{ }^{a}(\%)$ & $\eta_{\max }{ }^{b}$ & $R_{\eta}{ }^{b}\left(\mathrm{~s}^{-1}\right)$ \\
\hline EDB & 4.6 & 0.8 & 44.5 & 0 & 0 \\
TA & 3.1 & 1.2 & 52.0 & 0.32 & 0.025 \\
EDB-TA & 2.7 & 1.4 & 55.5 & 0.66 & 0.033 \\
I250 & 2.1 & 2.3 & 54.4 & 0.94 & 0.21 \\
EDB-I250 & 2.1 & 2.5 & 54.6 & $0.95(0.40)^{c}$ & 0.23
\end{tabular}

${ }^{a}$ Results obtained for homogeneous irradiation at $532 \mathrm{~nm}, 25 \mathrm{~mW} \mathrm{~cm}^{-2}$. ${ }^{b}$ Results obtained for inhomogeneous irradiation at $514 \mathrm{~nm}$ using holography setup, $25 \mathrm{~mW} \mathrm{~cm}^{-2}$. ${ }^{c}$ The value in brackets corresponds to the final diffraction efficiency.

a solid film is formed. This could be ascribed to the fast dechlorination reaction of $\mathrm{TA}^{-}$radical anions, formed after the photoinduced electron transfer between the excited dye and TA, ${ }^{20,21}$ which prevents any back electron transfer, thereby increasing the quantum yield of initiating radicals. Moreover, the EMP/TA system leads to the formation of a diffraction grating with a maximum rate of grating formation of about $0.025 \mathrm{~s}^{-1}$ and a maximum diffraction efficiency $\eta$ around $35 \%$.

According to the mechanistic studies, the three-component system EMP/EDB/TA is by far the most interesting. Using RT-FTIR, this system proved to be only slightly better with a final conversion around $55 \%$ and a rate of polymerization around $1.5 \mathrm{~s}^{-1}$ very close to that of EMP/TA. However, a high 

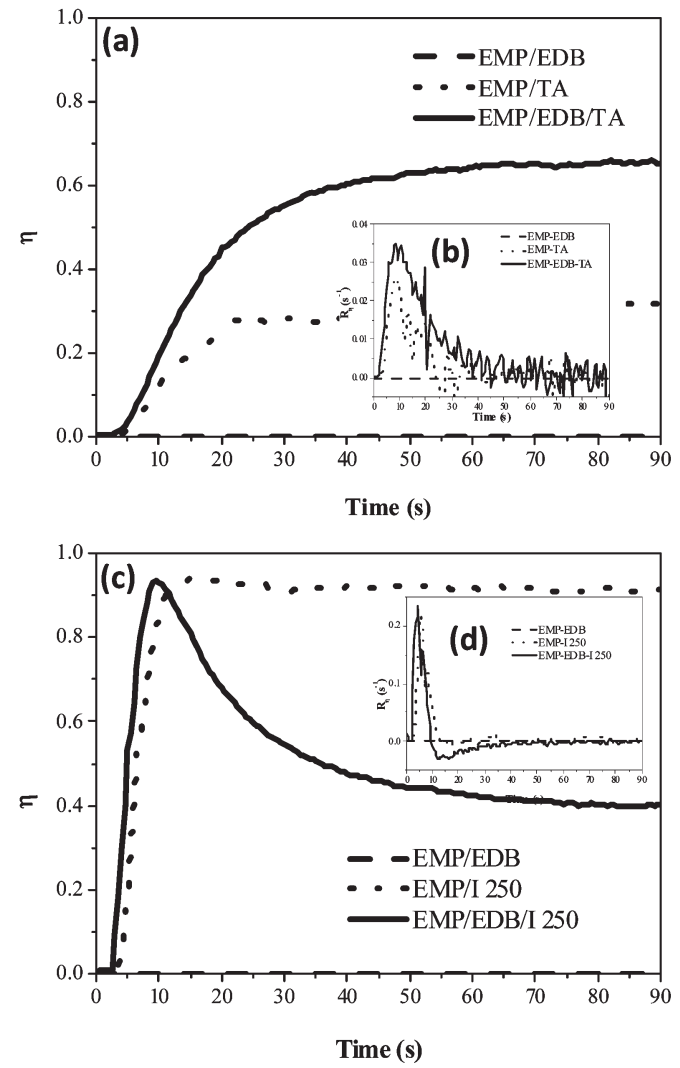

Fig. 3 Variation of the diffraction efficiency of the different photoinitiating systems as a function of time for an irradiance of $25 \mathrm{~mW} \mathrm{~cm}^{-2}$ at $514 \mathrm{~nm}$ and corresponding rate of grating formation (insets).

diffraction efficiency $\eta$ around $65-70 \%$ was noticed for this system with the highest maximum rate of grating formation $R_{\eta}$ around $0.033 \mathrm{~s}^{-1}$ (Fig. 3(b) right and Table 4). Such a high efficiency should be ascribed to the photocyclic reaction operating in the three component PIS. Thus, the PCIS based on EMP/ $\mathrm{EDB} / \mathrm{TA}$ clearly leads to the best holographic recording performances. This underlines how much holographic recording experiments reveal marked differences between the PIS that are not detected with RT-FTIR measurements. The difference in sensitivity between the two experiments could be ascribe to the particular mechanisms of grating formation, i.e. to the growing of the refractive index modulation due to a heterogeneous polymerization in the resin, compared to the homogeneous polymerization taking place in RT-FTIR measurements.

Indeed, under homogeneous irradiation of RT-FTIR experiments, it is known that the photopolymerization process can be so fast that the solidification of the medium occurs before full conversion. ${ }^{60,61}$ This is clearly observed for EMP/TA, EMP/ $\mathrm{EDB} / \mathrm{TA}, \mathrm{EMP} / \mathrm{I} 250$ and EMP/EDB/I250, with a maximum of conversion of about $50 \%$. No more driving force is available in homogeneous irradiation to push on the photopolymerization process, and the reaction is then stopped. It is worth noting that holographic recording behave differently. Indeed, the photopolymerization occurs rapidly in the exposed regions, but hardly proceeds in the unexposed regions. When the solidification takes place in the exposed regions, a gradient in the monomer concentration exists between the exposed and the unexposed regions.
This lead to a driving force which promotes the diffusion of the monomer from the unexposed regions to the exposed ones. ${ }^{62-64}$ Therefore, the photopolymerization reaction can continue as far as some photoinitiating system and monomer could be consumed. As in PCIS the dye is continuously recovered until full consumption of coinitiators, these PCIS systems appear to be much more efficient than two-component ones for grating formation. Then, fast and efficient photoinitiating systems result in the fast formation of refractive index modulation in the medium.

\section{EMP/EDB/I250 systems}

In order to improve the final diffraction efficiency of the previous system, which is limited to $66 \%$, the triazine derivative (TA) was replaced by an iodonium salt (I250), which exhibits a lower reduction potential. The photopolymerization kinetics of EMP/ EDB, EMP/I250 and EMP/EDB/I250 are shown in Fig. 2(b) and the most relevant data are collected in Table 4.

As can be seen, the two-component system based on EMP/ I250 exhibits good behaviour compared to the EMP/TA system. In particular the conversion rate is higher with a value around $2.3 \mathrm{~s}^{-1}$. Similar results were also found for the EMP/EDB/I250 system, with a high rate of conversion around $2.5 \mathrm{~s}^{-1}$ and a final conversion of about $55 \%$, very close to that of EMP/I250. Moreover, according to polymerization rates, iodonium based systems exhibit higher reactivity than those using TA. When looking only at RT-FTIR experiments, the PCIS based on I250 is not better than the corresponding two component PIS: no synergistic effect appears. Again, this levelling effect, attributed to the early solidification of the resin, looks to be in sharp contrast with the mechanistic studies where photocyclic behaviour was demonstrated.

Very interestingly, holographic recording experiments show quite different behaviour between the two and three component PIS based on I250. As can be seen in Fig. 3(c) (and Table 4) $\mathrm{EMP} / \mathrm{EDB} / \mathrm{I} 250$ is the fastest system. Notably, this photoinitiating system leads to a maximum diffraction efficiency $\eta$ close to unity, a value far higher than that of EMP/EDB/TA. Interestingly, the two-component system EMP/I250 also reaches a high diffraction efficiency $(\eta=0.94)$, albeit in a slightly longer time scale, i.e. using a higher dose than for EMP/EDB/I250 $\left(0.31 \mathrm{~J} \mathrm{~cm}^{-2}\right.$ instead of $0.22 \mathrm{~J} \mathrm{~cm}^{-2}$ for reaching the maximum diffraction efficiency). The difference in reactivity between the different photoinitiating systems are enhanced when using holographic recording compared to RT-FTIR experiments where final conversions are very close for the two PCIS, and where their conversion rates are only different by a factor less than two, outlining once again a levelling effect when using RT-FTIR.

\section{Comparison of PCIS based on I250 and TA}

For the sake of comparison, it is useful to extract the time needed to reach a given value of the diffraction efficiency $\eta$ for a given photoinitiating system. A $60 \%$ diffraction efficiency is reached for an irradiation time of $5.5 \mathrm{~s}$ with EMP/EDB/I250, whereas it required about $38 \mathrm{~s}$ when using the $\mathrm{EMP} / \mathrm{EDB} / \mathrm{TA}$ system. This means that the EMP/EDB/I250 system is about 7 times faster than EMP/EDB/TA. This is confirmed by the rate 
of hologram formation as $R_{\eta}$ was equal to $0.23 \mathrm{~s}^{-1}$ for EMP/ $\mathrm{EDB} / \mathrm{I} 250$ and $R_{\eta}=0.033 \mathrm{~s}^{-1}$ for EMP/EDB/TA.

It is worth noting that the behaviour of the three component $\mathrm{EMP} / \mathrm{EDB} / \mathrm{I} 250$ system is different to the others. Indeed, after a fast and efficient building up of the grating $\left(\eta_{\max }=95 \%\right)$, one can observe a decrease of its diffraction yield, which ends at a value of about $\eta_{\text {final }}=40 \%$ (Fig. 3(c)). Fig. 3(d) clearly evidences the fast growth of the hologram, with a diffraction rate $R_{\eta}$ reaching up to $0.23 \mathrm{~s}^{-1}$, followed by a negative rate of $-0.03 \mathrm{~s}^{-1}$. Four different hypotheses can be considered to explain the decrease of the material response: (1) subsequent decrease of the refractive index modulation after photopatterning has been recently observed and ascribed to the diffusion of some monomer type from exposed regions to unexposed regions due to chemical incompatibilities (solubilities) of the monomer with the formed polymer matrix; ${ }^{65}$ (2) lowering of $\eta$ related to the effects of an overmodulation of the refractive index inside the hologram; (3) the fast polymerization with EMP/EDB/I250 PCIS leading to non sinusoidal refractive index modulation; (4) the refractive index modulation partially erased in the last part of the grating building up due to polymerization in the dark fringes because of the very efficient PCIS.

Hypothesis (1) could not be retained in this work, as the liquid monomer mixture is the same for all the photopolymerizable experiment.

In the case of overmodulation, i.e. hypothesis (2), the refractive index modulation always continues to increase during the grating building up, but the diffraction efficiency decreases in the last part. According to the Kogelnik coupled-wave theory, with respect to the Bragg condition and considering here a transmissive dielectric grating (no absorption of the material at the reading wavelength), the diffraction efficiency is given by: ${ }^{66}$

$$
\eta=\sin ^{2}(v)
$$

where $v$ is the grating strength, which depends on the recording geometry and the photosensitive material (thickness, refractive index and modulation of the refractive index). Eqn (6) is able to justify the experimental results when $v$ becomes higher than $\pi / 2$. It is the case, for example, for the volume phase holograms recorded in mixtures of acrylamide and polyvinylalcohol in the presence of yellowish eosin and triethanolamine as a photosensitive system. ${ }^{58}$ In the present work, the angular response curves of the gratings demonstrated that overmodulation did not occur for these photopolymerizable systems: the shape of the curve of the grating diffraction efficiency at the end of the record as a function of the reconstructed angle did not point out a particular behaviour corresponding to a decrease of the diffraction efficiency in the central lobe associated with an increase in the lateral lobes. This rules out hypothesis (2). Moreover, as hypothesis (3) also leads to distorted angular response curves, which were not observed, this also rules out hypothesis (3).

In order to get more insights into this phenomenon, two additional experiments were performed by varying the irradiation conditions of the EMP/EDB/I250 system:

- First, the value of the incident irradiance used during holographic recording was decreased down to $4 \mathrm{~mW} \mathrm{~cm}^{-2}$. Fig. 4(a) and (b), respectively, show that high diffraction yields $\eta$ and maximum grating formation rate $R_{\eta}$ are obtained with a similar
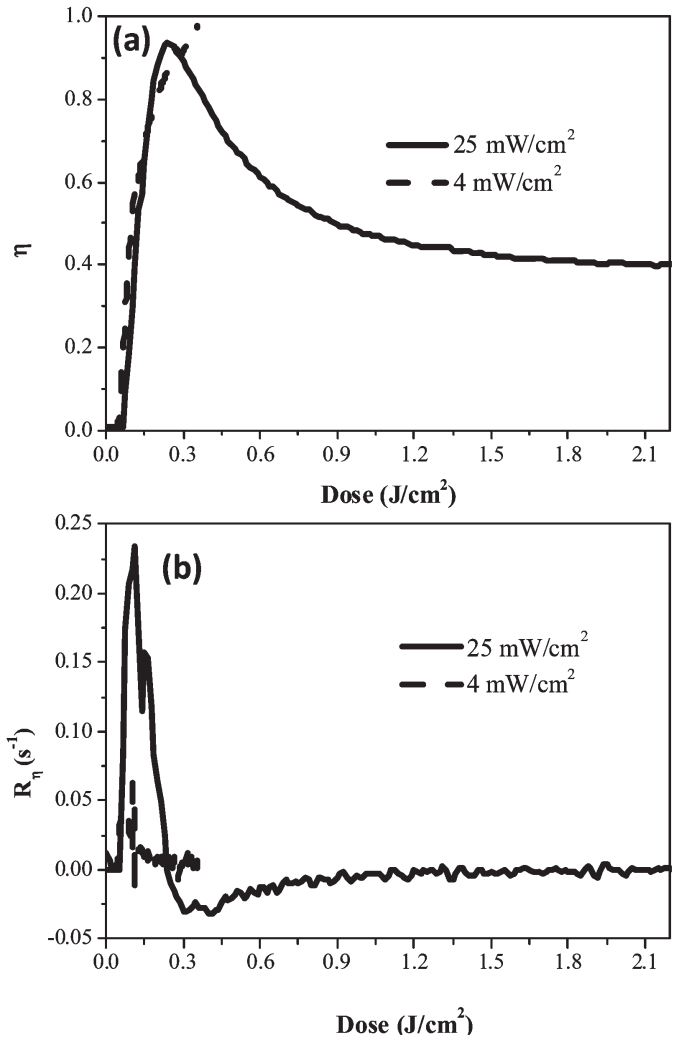

Fig. 4 Effect of irradiance intensity on (a) the diffraction efficiency $\eta$ curves and (b) the rate of grating formation $R_{\mathrm{h}}$ for the EMP/EDB/I250 PCIS; dotted lines: $4 \mathrm{~mW} \mathrm{~cm}^{-2}$; plain lines: $25 \mathrm{~mW} \mathrm{~cm}^{-2}$.

dose (around $0.3 \mathrm{~J} \mathrm{~cm}^{-2}$ ) for both 4 and $25 \mathrm{~mW} \mathrm{~cm}^{-2}$. The lack of intensity effect underlines the fact that the mass transfer rate of the monomer molecules during grating formation is not the cause of the final decrease of the refractive index modulation at $25 \mathrm{~mW} \mathrm{~cm}^{-2}$. At $4 \mathrm{~mW} \mathrm{~cm}^{-2}$, the rate of initiation is lower, corresponding to lower polymerization and grating formation rates, which lessen the influence of the mass transfer process. This result is also in agreement with angular response curves experiments, which allows the rejection of hypothesis (3).

- Secondly, the recording time was changed. It can be seen in Fig. 5(a) that, at $25 \mathrm{~mW} \mathrm{~cm}^{-2}$, a stable diffraction grating is obtained when the holographic exposure is switched off after $8 \mathrm{~s}$ of recording time. In the dark, the refractive index modulation is kept stable and does not decrease. It is also seen in Fig. 5(b) that in both experiments the maximum rates of grating formation are identical. These experiments highlight the fact that the grating destruction is a direct consequence of the continuous irradiation of the PCIS and, consequently, of the continuous production of radicals.

As all experiments were performed in the same conditions and in the same resin, the decrease of the grating efficiency $\eta$ is identified as a direct consequence of the chemistry of the photoinitiating system. Interestingly, it is the first time that such a result is reported. However, this experimentally observed decrease of the diffraction efficiency outlines the difference of response between RT-FTIR and diffraction measurements, as in RT-FTIR experiments both the conversion rate and the final conversion 

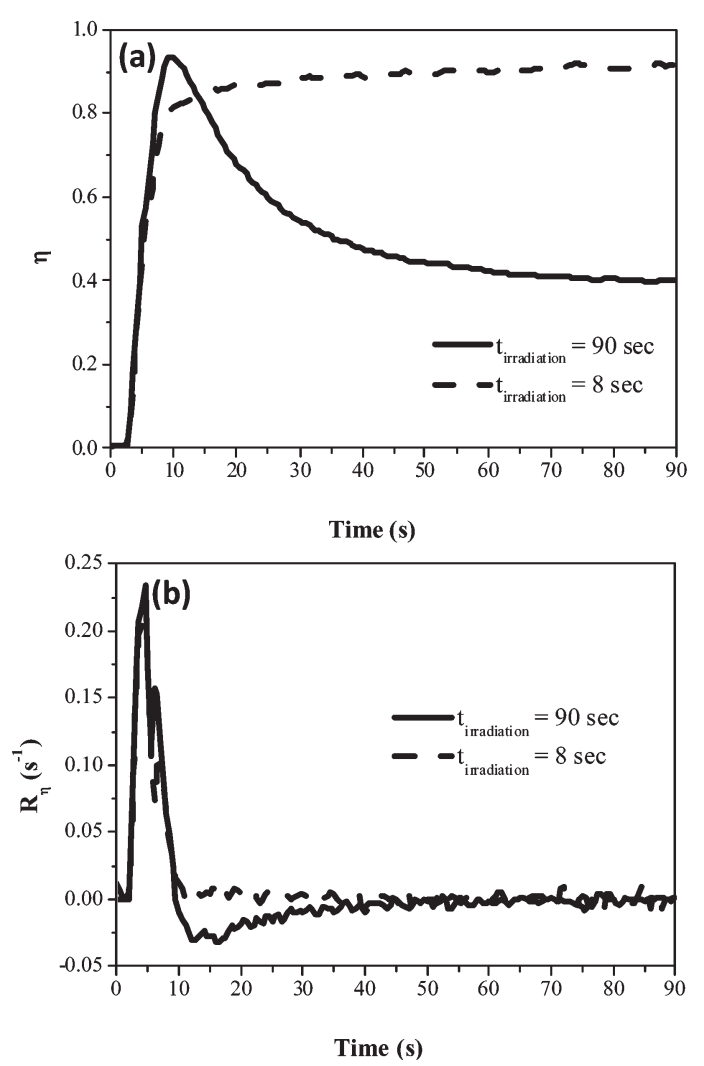

Fig. 5 Effect of the recording time on the diffraction efficiency of the grating $\left(25 \mathrm{~mW} \mathrm{~cm}^{-2}\right.$ at $\left.514 \mathrm{~nm}\right)$. The dotted line corresponds to an exposure duration of $8 \mathrm{~s}$ (after $8 \mathrm{~s}$, the $514 \mathrm{~nm}$ writing beam was switched off, keeping on the $633 \mathrm{~nm}$ reading beam).

degree are similar for EMP/I250 and EMP/EDB/I250 systems. Furthermore, the final monomer conversion degrees reach the same plateau value for longer irradiation times.

Taking into account all the previous experiments, the modulation of refractive index can be rationalized as a consequence of the high efficiency of the photocyclic behaviour of the EMP/ EDB/I250 photoinitiating system. Indeed, it was shown that three-component systems lead to efficient recovery of the dye during the photocycle. As explained in the mechanistic studies of the paper, the dye concentration should be constant, at least staying high as long as the coinitiators are both present, leading to a continuous production of initiating radicals. In the bright areas, this species consumes the oxygen present in the medium and initiates the polymerization reaction. Then an overflow of radicals, which can diffuse into dark fringes, occurs and starts the polymerization in the dark fringes, which induces the destruction of the refractive index modulation.

Furthermore, in the dark areas the initiation of the polymerization is first stopped by oxygen inhibition as the density of incident photons is low. However, after a certain exposure time, the continuous formation of radicals of the EMP/EDB/I250 PCIS will consume all the dissolved oxygen in the dark areas and give rise to polymerization. As a result of the polymerization taking place slowly in the regions of lowest irradiance, the refractive index modulation decreases between dark and bright areas during the recording process and the diffraction efficiency decreases.

\section{Conclusions}

In this work, we used a photopolymerizable resin, optimized as an efficient holographic recording medium, to probe the reactivity of photocyclic initiating systems (PCIS) based on a pyrromethene dye (EMP), an amine as an electron donor, and an electron acceptor. It is shown that high diffraction efficiencies are achieved, underlining that the considered medium would be suitable for WORM (write once read many) applications. Holographic recording has revealed the particular behaviour of PCIS and reveals marked differences between the PIS that are not detected with classical RT-FTIR measurements. Further works would focus on an evaluation of the yield of the photocyclic reaction depending on the experimental conditions.

\section{Notes and references}

1 R. Schwalm, UV Coatings, Basics, Recent Developments and New Applications, Elsevier, Amsterdam, 2007.

2 X. Allonas, C. Croutxe-Barghorn, J. P. Fouassier, J. Lalevee, J. P. Malval and F. Morlet-Savary, in Lasers in Chemistry. Influencing Matter, ed. M. Lackner, Wiley, Weinheim, 2008, ch. 35, vol. 2, p. 1001.

3 W. Schnabel, Polymers and Light: Fundamentals and Technical Applications, 2007, p. 396.

4 K. Curtis, L. Dhar and W. L. Wilson, Comprehensive Nanoscience and Technology, Elsevier, 2011, vol. 4, pp. 615-630.

5 S. Gallego, A. Márquez, M. Ortuño, S. Marini and J. Francés, Opt. Mater., 2011, 33, 1626-1629.

6 SPIE's Holography Newsletter, Special Issue on: Holographic Materials for Data Storage, 2003, 14(2).

7 M. M. Wang and S. C. Esener, Appl. Opt., 2000, 39, 1826-1834.

8 W. J. Joo, C.-H. Oh, S.-H. Song, P.-S. Kim and Y.-K. Han, J. Phys. Chem. B, 2001, 105, 8322-8326.

9 U.-S. Rhee, H. J. Caulfield, C. S. Vikram and J. Shamir, Appl. Opt., 1995, 34, 846-853.

10 N. Suzuki, Y. Tomita and T. Kojima, Appl. Phys. Lett., 2002, 81, $4121-4123$.

11 T. J. Trentler, J. E. Boyd and V. L. Colvin, Chem. Mater, 2000, 12, 1431-1438.

12 Y. Takamatsu, D. Dunmeyer, E. L. Thomas and C. Warde, Opt. Lett., 2008, 33, 7-9.

13 E. Leite, I. Naydenova, S. Mintova, L. Leclercq and V. Toal, Appl. Opt., 2010, 49, 3652-3660.

14 K. Chikama, K. Mastubara, S. Oyama and Y. Tomita, J. Appl. Phys., 2008, 103, 113108 .

15 L. Hesselink, S. S. Orlov and M. C. Bashaw, Proc. IEEE, 2004, 92, 1231-1280.

16 Y. Bi and D. C. Neckers, J. Photochem. Photobiol., A, 1993, 74, 221-230.

17 D. F. Eaton, Top. Curr. Chem., 1990, 156, 199-225.

18 T. Urano, E. Hino, H. Ito, M. Shimizu and T. Yamaoka, Polym. Adv. Technol., 1998, 9, 825-830.

19 X. Allonas, J. P. Fouassier, M. Kaji, M. Miyasaka and T. Hidaka, Polymer, 2001, 42, 7627-7634.

20 A. Ibrahim, C. Ley, O. I. Tarzi, J. P. Fouassier and X. Allonas, J. Photopolym. Sci. Technol., 2010, 23, 101-108.

21 O. I. Tarzi, X. Allonas, C. Ley and J. P. Fouassier, J. Polym. Sci., Part A: Polym. Chem., 2010, 48, 2594-2603.

22 J. Karolin, L. B. A. Johansson, L. Strandberg and T. Ny, J. Am. Chem. Soc., 1994, 116, 7801-7806.

23 F. Li, S. I. Yang, Y. Ciringh, J. Seth, C. H. Martin, D. L. Singh, D. Kim, R. R. Birge, D. F. Bocian, D. Holten and J. S. Lindsey, J. Am. Chem. Soc., 1998, 120, 10001-10017.

24 P. Toele, H. Zhang, C. Trieflinger, J. Daub and M. Glasbeek, Chem. Phys. Lett., 2003, 368, 66-75.

25 J. Bañuelos Prieto, F. López Arbeloa, V. Martínez Martínez, T. Arbeola López, F. Amat-Guerri, M. Liras and I. López Arbeloa, Chem. Phys. Lett., 2004, 385, 29-35.

26 K. Jagtap, D. Maity, A. Ray, K. Dasgupta and S. Ghosh, Pramana, 2010, 75, 985-989. 
27 A. Costela, I. García-Moreno, C. Gomez, R. Sastre, F. Amat-Guerri, M. Liras, F. López Arbeloa, J. Bañuelos Prieto and I. López Arbeloa, J. Phys. Chem. A, 2002, 106, 7736-7742.

28 S. Suzuki, X. Allonas, J.-P. Fouassier, T. Urano, S. Takahara and T. Yamaoka, J. Photochem. Photobiol., A, 2006, 181, 60-66.

29 A. A. Gorman, I. Hamblett, T. A. King and M. D. Rahn, J. Photochem. Photobiol., A, 2000, 130, 127-132.

30 G. Jones, S. Kumar, O. Klueva and D. Pacheco, J. Phys. Chem. A, 2003, 107, 8429-8434.

31 T. G. Pavlopoulos, J. H. Boyer, M. Shah, K. Thangaraj and M.-L. Soong, Appl. Opt., 1990, 29, 3885-3886.

32 F. López Arbeloa, T. López Arbeloa, I. López Arbeloa, I. García-Moreno, A. Costela, R. Sastre and F. Amat-Guerri, Chem. Phys., 1998, 236, 331-341.

33 C. Angel, G.-M. Inmaculada, G. Clara, G. Olga and S. Roberto, Chem. Phys. Lett., 2003, 369, 656-661.

34 M. S. Mackey and W. N. Sisk, Dyes Pigm., 2001, 51, 79-85.

35 M. Ahmad, T. A. King, D.-K. Ko, B. Heon Cha and J. Lee, Opt. Commun., 2002, 203, 327-334.

36 M. D. Rahn, T. A. King, A. A. Gorman and I. Hamblett, Appl. Opt., 1997, 36, 5862-5871.

37 N. Tanaka and W. N. Sisk, J. Photochem. Photobiol., A, 2005, 172, 109-114.

38 G. Beer, C. Niederalt, S. Grimme and J. Daub, Angew. Chem., Int. Ed., $2000,39,3252-3255$.

39 K. Rurack, M. Kollmannsberger and J. Daub, Angew. Chem., Int. Ed., 2001, 40, 385-387.

40 T. Arbeola López, F. López Arbeloa, I. López Arbeloa, I. García-Moreno, A. Costela, R. Sastre and F. Amat-Guerri, Chem. Phys. Lett., 1999, 299, 315-321.

41 R. E. Hermes, T. H. Allik, S. Chandra and J. A. Hutchinson, Appl. Phys. Lett., 1993, 63, 877-879.

42 T. Urano, E. Ohno-Okumura, K. Sakamoto, H. Ito and T. Yamaoka, J. Photopolym. Sci. Technol., 1999, 12, 747-757.

43 M. Kollmannsberger, K. Rurack, U. Resch-Genger and J. R. Daub, J. Phys. Chem. A, 1998, 102, 10211-10220.

44 F. Bergström, I. Mikhalyov, P. Hägglöf, R. Wortmann, T. Ny and L. B.-A. Johansson, J. Am. Chem. Soc., 2001, 124, 196-204.
45 S. Noppakundilograt, S. Suzuki, T. Urano, N. Miyagawa, S. Takahara and T. Yamaoka, Polym. Adv. Technol., 2002, 13, 527-533.

46 T. Urano, H. Ito and T. Yamaoka, Polym. Adv. Technol., 1999, 10, 321-328.

47 S. Blaya, P. Acebal, L. Carretero and A. Fimia, Opt. Commun., 2003, 228, 55-61.

48 M. M. Wang and S. C. Esener, Appl. Opt., 2000, 39, 1826-1834.

49 M. De Sarkar, J. Qi and G. P. Crawford, Polymer, 2002, 43, 7335-7344.

50 M. D. Schulte, S. J. Clarson, L. V. Natarajan, D. W. Tomlin and T. J. Bunning, Liq. Cryst., 2000, 27, 467-475.

51 T. M. Roper, T. Kwee, T. Y. Lee, C. A. Guymon and C. E. Hoyle, Polymer, 2004, 45, 2921-2929.

52 C. Carre, R. Chevallier, B. Mailhot and A. Rivaton, Basics and Applications of Photopolymerization Reactions, ed. J. P. Fouassier and X. Allonas, Research Signpost, Trivandrum, India, 2010, vol. 3, pp. $175-184$

53 S. Massenot, R. Chevallier, J.-L. de Bougrenet de la Tocnaye and O. Parriaux, Opt. Commun., 2007, 275, 318-323.

54 P. Jacques, D. Burget and X. Allonas, New J. Chem., 1996, 30, 933.

55 C. Decker and K. Moussa, Macromolecules, 1989, 22, 4455-4462.

56 X. Allonas, J. P. Fouassier, L. Angiolini and D. Caretti, Helv. Chim. Acta, 2001, 84, 2577-2588.

57 S. Massenot, J. L. Kaiser, R. Chevallier and Y. Renotte, Appl. Opt., 2004, 43, 5489-5497.

58 S. Gallego, M. Ortuno, C. Neipp, C. Garcia, A. Beléndez and I. Pascual, Opt. Commun., 2003, 215, 263-269.

59 D. Rehm and A. Weller, Isr. J. Chem., 1970, 8, 259.

60 C. Decker, Prog. Polym. Sci., 1996, 21, 593-650.

61 C. Decker, Macromol. Rapid Commun., 2002, 23, 1067-1093.

62 J. R. Lawrence, F. T. O’Neill and J. T. Sheridan, Optik, 2001, 112, 449.

63 M. R. Gleeson and J. T. Sheridan, J. Opt. A: Pure Appl. Opt., 2009, 11, 024008.

64 M. Květoň, A. Havránek, P. Fiala and I. Richter, Polym. Bull., 2007, 58, 253.

65 S. Han, M. Lee and B. K. Kim, Opt. Mater, 2011, 34, 131-137.

66 H. Kogelnik, Bell Syst. Tech. J., 1969, 48, 2909-2947. 